\section{Eculizumab for complement mediated thrombotic microangiopathy in sickle cell disease}

Despite being the first genetic disease described, sickle cell disease (SCD) continues to afflict patients with immense pain, significant comorbidities and premature death. SCD has only recently benefited from new interventions with L-glutamine (2017), voxelotor (2019) and crizanlizumab (2019) representing the first Food and Drug Administration approved medications for SCD since hydroxyurea in 1997. These interventions have demonstrated some ability to reduce vaso-occlusive pain crisis episodes, improve hemoglobin (HGB), or reduce markers of hemolysis and have largely been used as preventative care measures. While these and additional approaches, such as hematopoietic stem cell transplant and gene therapy, can improve SCD care, many patients with SCD continue to suffer from severe acute SCD complications that can result in organ damage and early death. ${ }^{1,2}$ Unfortunately, in these situations, supportive care remains the primary approach to alleviate complications. The lack of more targeted approaches in part reflects an incomplete understanding of the pathophysiology and accompanying pharmacological targets that could specifically mitigate acute disease complications. We present a summary of three cases of children with SCD who developed significant acute complications that demonstrate underlying complement-mediated thrombotic microangiopathy (CM-TMA). These cases include a delayed hemolytic transfusion reaction (DHTR), vasoocclusive crisis (VOC) and drug-induced immune hemolytic anemia (DIIHA).

Patient \#1 is a 14-year-old male with a history of two episodes of DHTR. At the age of 12 years, he received a transfusion pre-operatively for hip core-decompression. He presented 7 days later with severe diffuse body pain, hemoglobinuria, fever, and total HGB of $9.4 \mathrm{~g} / \mathrm{dL}$ with hemoglobin $\mathrm{A}(\mathrm{HbA})$ at $17 \%$ (Figure $1 \mathrm{~A})$. Further testing revealed a previously detected anti-U alloantibody, negative direct antiglobulin test (DAT) and evidence for intravascular hemolysis. On the night of admission, he became hypertensive with headache and sluggish mentation. A brain magnetic resonance imaging scan was normal. HGB dropped to $5.6 \mathrm{~g} / \mathrm{dL}$ within 30 hours of admission, and the patient rapidly deteriorated to multiorgan failure (MOF) with thrombocytopenia (Figure 1A). Due to strong suspicion for DHTR with hyperhemolysis and CM-TMA, he was treated with eculizumab $600 \mathrm{mg}$ intravenously (IV) and erythropoietin $150 \mathrm{IU} / \mathrm{kg}$ to augment erythropoiesis. Over the next 24 hours, he developed a new consolidation in the left lung consistent with acute chest syndrome (ACS). He received one unit of crossmatch compatible, U-negative red blood cells (RBC) after a dose of $1 \mathrm{~g} / \mathrm{kg}$ intravenous immunoglobulin (IVIG) on day 9. Over the next few days, he made a gradual improvement in clinical and laboratory status. Eculizumab $600 \mathrm{mg}$ was continued weekly for a total of four doses. Subsequent analysis revealed significant alternative complement pathway (ACP) activation at the peak

Table 1. Evaluation of complement pathway during acute sickle cell crises.

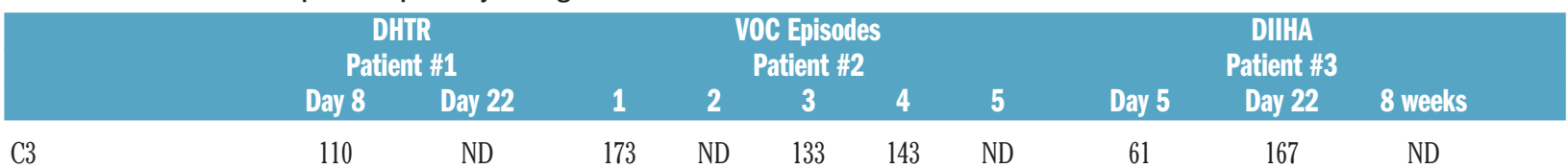

(71-150 mg/dL)

$\begin{array}{llllllllllll}\text { C4 } & 14.2 & \text { ND } & 25 & \text { ND } & 21.6 & 21 & \text { ND } & 6 & 26 & \text { ND } & \\ (15.7-47 \mathrm{mg} / \mathrm{dL}) & 362 & \text { ND } & \text { ND } & \text { ND } & \text { ND } & \text { ND } & 260 & 30 & 4 & \text { ND } & \end{array}$

(101-300 units)

$\begin{array}{lccccccccccc}\mathrm{Bb} & 9.75 & 1.41 & 1.9 & 1.2 & 1.3 & 1.3 & 1.9 & 23.6^{*} & 1.63 & 2.185 & \\ (0.49-1.42 \mathrm{mcg} / \mathrm{mL})^{*} & & & & & & & & & & & \\ \mathrm{C} 3 \mathrm{a} & 375.5 & 127.2 & 124 & 86 & 76.4 & 115 & 164 & 210.4 & 102.5 & 182.9 & \end{array}$

(25-88.2 $\mathrm{ng} / \mathrm{mL})$

$\begin{array}{lccccccccccc}\text { C5a } & >37.3 & 31.26 & 20 & 23 & 13.5 & 17.2 & 26 & >38.0 & 35.53 & 27.6 & \\ (2.74-16.33 \mathrm{ng} / \mathrm{mL}) & 1208 & 307 & 97 & 145 & 112 & 153 & 83 & >1800 & 554 & 394\end{array}$

$(\leq 244 \mathrm{ng} / \mathrm{mL})$

$\begin{array}{lcccccc}\begin{array}{l}\text { Day of eculizumab } \\ \text { administration }\end{array} & \begin{array}{c}\text { Days } 8,15,22 \\ \text { and } 29\end{array} & \text { None } & \text { None } & \begin{array}{c}\text { same } \\ \text { day }\end{array} & \begin{array}{c}\text { same } \\ \text { day }\end{array} & \begin{array}{c}\text { none } \\ \text { every } 2 \text { weeks* }\end{array}\end{array}$

DHTR: delayed hemolytic transfusion reaction; VOC: vaso-occlusive crisis, DIIHA: drug induced immune hemolytic anemia; C3: complement component 3; C4: complement component 4; CH50: screening test for total complement activity; Bb: complement component fragment Bb; C3a: complement component fragment 3a; C5a: complement component fragment 5a; sC5b-9: soluble membrane attack complex; ND: not done. *8-week laboratory analysis were performed few hours prior to eculizumab administration. The whole blood sample for all testing was collected in EDTA anticoagulant tubes and plasma was obtained within 2 hours of collection, and stored in $-80^{\circ} \mathrm{C}$ until they were ready for analysis with single thawing. This method of plasma collection in EDTA results in chelation of calcium and magnesium thus preventing any in vitro complement activation. All testing was obtained in a Clinical Laboratory Improvement Amendments (CLIA) certified hospital-based clinical laboratory. All normal values are in parentheses under each value except in patient $\# 3$, day $5 *$ Bb normal ranges were $1.32-4.18 \mathrm{mcg} / \mathrm{mL}$ due to variability seen with different enzyme-linked immunosor bent assay kits. Patient \#1, day 8 and day 22 signifies the complement evaluation a few hours prior to respective dosing of eculizumab.Patient\#2 presented with five episodes of VOC (each column represents episodes 1 through 5) with complement function testing coinciding with a drop in hemoglobin of greater than 2 g/dL from baseline. Eculizumab were dosed a few hours after samples were collected during episodes \#3 and \#5. Patient \#3, days 5 , 22 and 8 weeks reflect the complement evaluation. Complement proteins C3 and C4 signify the quantitative serum levels. Fragment Bb is a serine protease that in combination with hydrolyzed C3 (C3H2O) generates C3 convertase $(\mathrm{C} 3 \mathrm{bBb}$ ), which augments the cleavage of $\mathrm{C} 3$ to generate $\mathrm{C} 3 \mathrm{a}$ and $\mathrm{C} 3 \mathrm{~b}$. Anaphylatoxins, $\mathrm{C} 3 \mathrm{a}$ and $\mathrm{C} 5 \mathrm{a}$ are involved in local inflammation and tissue damage, while C3b results in red blood cell opsonization and deposition on endothelium. Terminal complex, C5b-9 contributes to intravascular hemolysis. 
A

Patient \#1
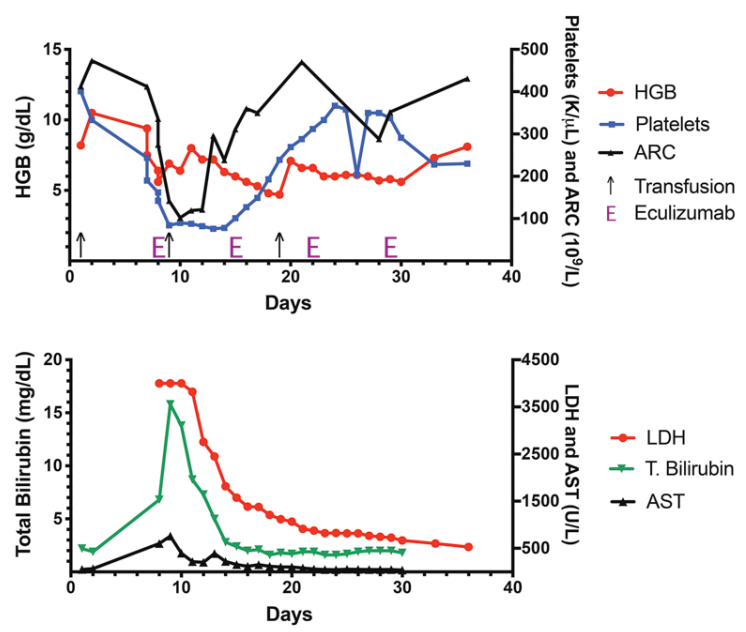

C

Patient \#3

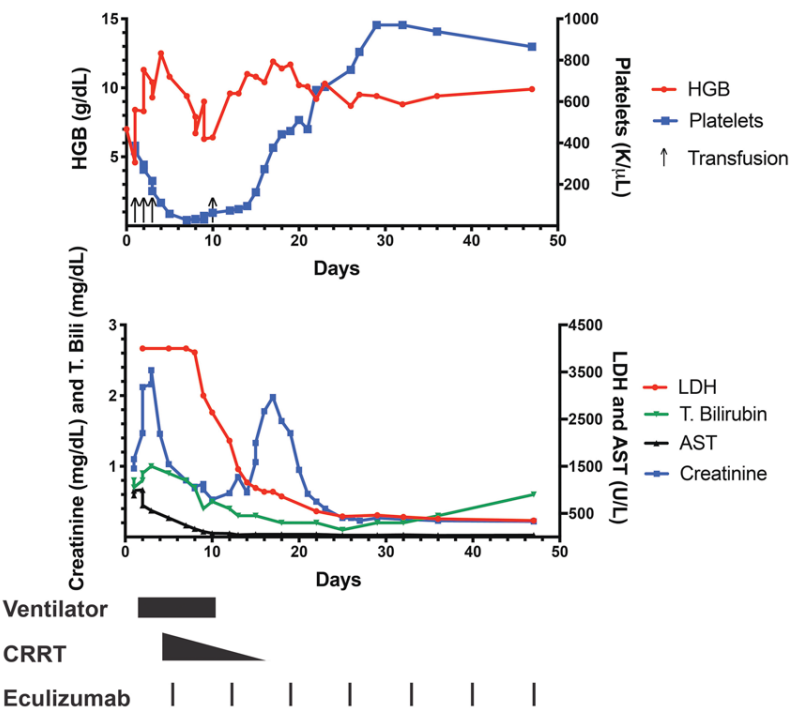

B
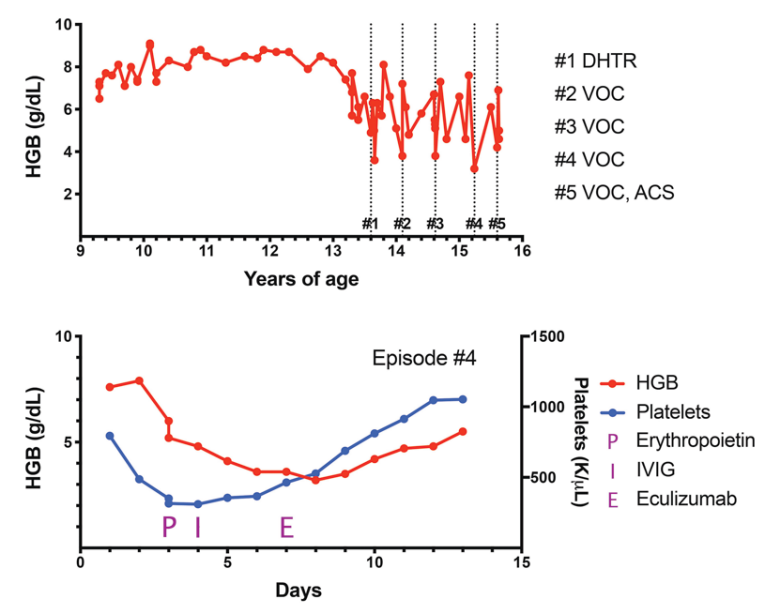

D

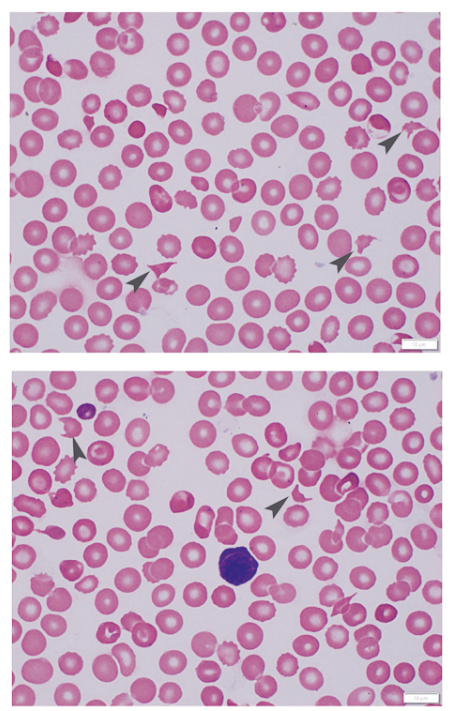

Figure 1. A graphic representation of a delayed hemolytic transfusion reaction, vaso-occlusive crisis and drug induced immune hemolytic anemia episode. (1A) DHTR (delayed hemolytic transfusion reaction): the patient received a transfusion (black arrows) on day one and presented with DHTR on day 7 with a hemoglobin (HGB) of $9.4 \mathrm{~g} / \mathrm{dL}$, which dropped to a nadir of $5.6 \mathrm{~g} / \mathrm{dL}$ by day 8 along with absolute reticulocytopenia, as expected in DHTR. Thrombocytopenia coincided with this severe anemia and elevations in lactate dehydrogenase (LDH), total bilirubin (T.Bili), and aspartate transaminase (AST). Other evidence for intravascular hemolysis included reduced haptoglobin (<14; reference 30-120 mg/dL) and elevated plasma free HGB (120; reference < $30 \mathrm{mg} / \mathrm{dL})$ not shown. Eculizumab (E) $600 \mathrm{mg}$ was initiated on day 8 and given weekly for a total of four doses. The patient developed acute chest syndrome (ACS) and received one unit of crossmatch compatible, U-negative red blood cells (RBC) after a dose of intravenous immunoglobulin (IVIG) on day 9. The resolution of hemolysis was evidenced by improvement in LDH and HGB following the first dose of eculizumab and maintained throughout the hospital stay. Thrombocytopenia recovered within 1 week of complement inhibition. The patient required an additional transfusion on day 19 from rebound anemia likely secondary to frequent blood draws, since markers of hemolysis and complement activation on day 22 did not worsen. Immunohematology work-up during this DHTR episode: direct antiglobulin test (DAT) negative, historical anti-U detected. Note: the initial three LDH values were greater than 4,000 U/L (upper limit of our clinical laboratory detection). Eculizumab was dosed based on the weight of the patient, as per the guidelines for loading dose for children with atypical hemolytic uremic syndrome (aHUS). (1B) Vaso-occlusive crisis (VOC): grey colored dotted bars depict the time points when the patient presented with VOC and also corresponds to complement testing (numbered \#1 through \#5). Eculizumab was administered for episodes \#3 and \#4 as described. Lower graph represents episode \#4 of VOC and shows an initial drop in HGB and platelets by day 4, when erythropoietin (P) $210 \mathrm{IU} / \mathrm{kg}$ was commenced along with a dose of IVIG (I) $1 \mathrm{~g} / \mathrm{kg}$ on days 4 and 5 , respectively. Given the continued deterioration in HGB to a nadir of $3.6 \mathrm{~g} / \mathrm{dL}$ along with severe headache and worsening hypoxia, eculizumab (E) 900 mg was administered on day 7 after the blood for complement function analyses was collected. Rapid improvement in hemolysis and clinical status was observed within 48 hours of a single eculizumab dose. Eculizumab was dosed based on the weight of the patient, as per the guidelines for loading dose for children with aHUS. (1C) Drug induced immune hemolytic anemia (DIIHA): the patient presented on day 0 to the hospital with fever and received ceftriaxone; HGB dropped within 3 hours to $4 \mathrm{~g} / \mathrm{dL}$. Black arrows on the graph denote blood transfusions. Additional laboratory workup showed evidence of intravascular hemolysis, confirmed by elevated $\mathrm{LDH}>4,000 \mathrm{U} / \mathrm{L}$, presence of schistocytes on blood smear and elevated plasma free HGB (not shown). Multiorgan failure was evident with a peak of creatinine at $2.36 \mathrm{mg} / \mathrm{dL}$ (baseline $0.3 \mathrm{mg} / \mathrm{dL}$ ), and AST/alanine transaminase at 1,002/70 U/L along with rise in T.Bili. Black colored bars at the bottom of the graph depict the time points when various supportive care measures and eculizumab were administered. Shortly after the initiation of eculizumab, hemolysis decreased, as shown by the rapid drop in LDH, and the patient required less transfusion support. Thrombocytopenia improved. He had initial improvement in creatinine on continuous renal replacement therapy (CRRT), with a brief increase when CRRT was weaned. This rise was not sustained, and creatinine levels decreased promptly without any additional intervention except continued eculizumab therapy. By day 25 , blood counts and chemistry were within normal limits. The rebound thrombocytosis persisted for few weeks before trending back to the patient's baseline. Note: the initial three LDH values were greater than 4,000 $\mathrm{U} / \mathrm{L}$ (upper limit of our clinical laboratory detection). Eculizumab was dosed based on the weight of the patient, as per the guidelines for loading dose for children with aHUS. Blood smears from days 4 and 5 showing the presence of schistocytes and helmet cells (black arrows), along with paucity of platelets. ARC: absolute reticulocyte count. 


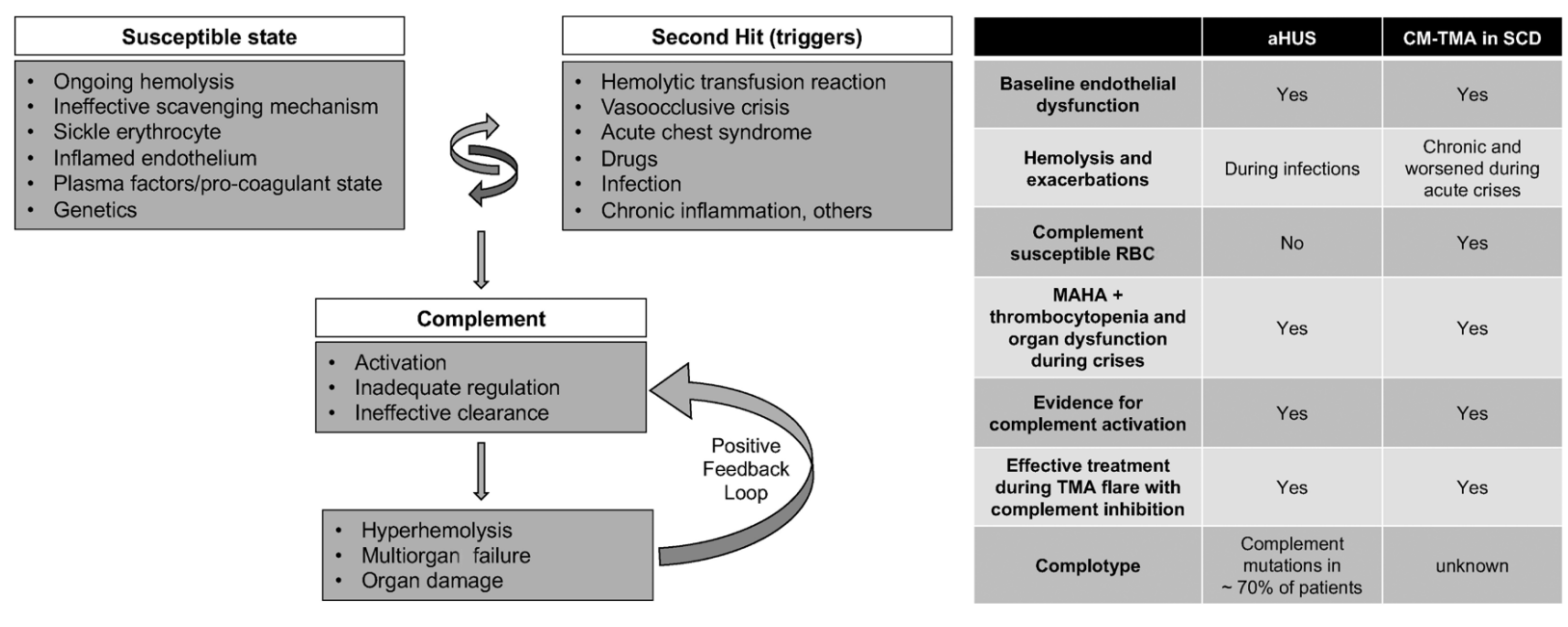

Figure 2. A model for complement- mediated thrombotic microangiopathy in sickle cell disease. Increased understanding of complement-mediated conditions such as atypical hemolytic uremic syndrome (aHUS) and paroxysmal nocturnal hemoglobinuria has renewed interest in understanding the specific role of complement in hyperhemolysis and innate immunity. Sickle cell disease (SCD) is a prototypical disease of chronic hemolysis, where increased levels of free plasma hemoglobin and heme with an insufficient, and thus ineffective scavenging mechanism by haptoglobin and hemopexin, leads to a state that is primed for complement activation. In addition, sickle erythrocytes themselves appear to be uniquely susceptible to complement-induced hemolysis, thereby further amplifying complement activation and additional hemolysis. Like in aHUS, the already inflamed endothelium in patients with SCD can be further modulated from increased hemolysis, complement activation, coagulation dysfunction, and other plasma proteins. Further genetic studies focusing on complotype are needed to help understand its role in SCD, as they can modulate the homeostatic balance of complement activity. Triggers such as pain crisis, acute chest syndrome, infection, etc. can drive this vulnerable state very quickly into a complement activated state, which, if unregulated, can result in common and terminal complement pathway activation that can lead to catastrophic damage in end organs and even death. Irrespective of the instigating cause, once complement-mediated hyperhemolysis is set off, it could result in a positive feedback loop causing further complement activation and a precipitous drop in hemoglobin and risk of sudden death. The table on the right parallels the resting and enabling state seen in SCD to patients with aHUS. CM-TMA: complement-mediated thrombotic microangiopathy; MAHA: microangiopathic hemolytic anemia; RBC: red blood cell.

of hemolysis, as evidenced by increased complement component fragment $\mathrm{Bb}(\mathrm{Bb})$ levels, anaphylatoxins (C3a and $\mathrm{C} 5 \mathrm{a})$ and terminal complement complex (C5b-9) (Table 1). Testing for complement regulatory genes $(C F H$, CFI, MCP (CD46), CFB, CFHR5, C3, THBD, DGKE, PLG, $A D A M T S 13$ and $M M A C H C$ ) revealed a homozygous deletion of complement factor H-related protein (CFHR) 3 and CFHR1, but criteria for DEAP-HUS (deficiency of CFHR plasma proteins and autoantibody positive form of HUS) were not met due to the absence of factor $\mathrm{H}$ autoantibodies.

Patient \#2 is a 15-year-old female with a history of VOC episodes and DHTR, who was transferred to our care at the age of 9 years. Since transfer, she suffered from multiple episodes of VOC, each accompanied with a drop in HGB. Five of these episodes are shown in the dotted lines (Figure 1B, top). During episode \#3, with no recent $\mathrm{RBC}$ transfusion history, the patient presented with an HGB of $6.7 \mathrm{~g} / \mathrm{dL}$ which decreased to $3.8 \mathrm{~g} / \mathrm{dL}$ over 5 days, accompanied by lethargy, hypoxia and respiratory distress. Given her past history of DHTR and rapid decompensation, one dose of eculizumab $900 \mathrm{mg}$ IV was administered along with erythropoietin $210 \mathrm{IU} / \mathrm{kg}$ daily and IVIG at $1 \mathrm{~g} / \mathrm{kg}$. The patient's symptoms resolved with a rise in HGB and she was discharged home within 4 days. Six months later, she presented again (\#4) with VOC, worsening hypoxia, and HGB $7.9 \mathrm{~g} / \mathrm{dL}$ (Figure 1B, bottom). On admission, she received erythropoietin and IVIG at the doses outlined above. The HGB dropped to a nadir of $3.6 \mathrm{~g} / \mathrm{dL}$ over 3 days, associated with severe headaches. Due to inadequate response to the above measures, eculizumab $900 \mathrm{mg}$ was administered with rapid improvement in 48 hours. Complement analyses (Table 1) indicated clear evidence of ACP activation during episodes \#1 and \#5, as shown by elevation of $\mathrm{Bb}$ le- vels. During episodes \#3 and \#4, eculizumab was administered in anticipation of worsening organ function, which could explain why the complement activation markers were not significantly elevated (unlike in other episodes) and why her rapid $\mathrm{HGB}$ response and prompt reversal of organ dysfunction were observed. Complement gene evaluation revealed a heterozygous deletion of CFHR3/CFHR1.

Patient \#3 is a 3 -year-old male with a history of splenectomy at the age of 2 years for recurrent acute splenic sequestration. He presented with fever, tachypnea, HGB of $7 \mathrm{~g} / \mathrm{dL}$, with a negative chest x-ray (CXR). He developed increased work of breathing within 3 hours of receiving ceftriaxone. Repeat CXR revealed bilateral infiltrates requiring emergent intubation. HGB dropped to $4 \mathrm{~g} / \mathrm{dL}$, and he received extended phenotypematched and crossmatch-compatible RBC transfusions. Additional laboratory work-up revealed intravascular hemolysis and MOF (Figure 1C). The DAT was positive for complement component 3 (C3) only. The patient required escalation of respiratory support to high frequency oscillatory ventilation and nitric oxide. Oliguric acute kidney injury and hypertension required the use of continuous renal replacement therapy and multiple antihypertensives. Multiple common and rare causes for his rapid multi-organ failure were entertained including sepsis (negative blood/respiratory cultures), cold agglutinin syndrome (negative testing for mycoplasma serology and Donath-Landsteiner antibody), hemophagocytic lymphohistiocytosis (normal soluble interleukin-2 receptor, CD107a, perforin/granzyme B, Epstein Barr and cytomegaloviral load) and ceftriaxone-induced hemolysis. As his clinical course was consistent with CM-TMA, complement inhibition with eculizumab $600 \mathrm{mg}$ IV was initiated on day 5 . Following eculizumab, he demonstrat- 
ed a rapid response with weaning of his ventilator support and dialysis, along with the reduced need for blood products. Follow-up testing was notable for strongly positive ceftriaxone-dependent antibodies consistent with DIIHA. Complement analyses confirmed significant activation of ACP (factor Bb elevation, see Table 1). Proximal and terminal complement pathway activation were likewise observed as indicated by increased C3 and C5 activation and C5b-9, respectively. Additionally, hypocomplementemia with reduced $\mathrm{C} 3, \mathrm{C} 4$, and $\mathrm{CH} 50$, seen in this patient, suggests worse disease. These markers improved following complement inhibition, which correlated with improved clinical status within 11 days of initial presentation. Eight months after this episode, this patient's renal function is gradually improving. $\mathrm{He}$ remains on eculizumab $300 \mathrm{mg}$ every 2 weeks pending full renal recovery. Complement genetic analysis was negative.

All patients and/or their guardians of cases described in this report provided written consent for the off-label use of eculizumab. These patients received meningococcal and pneumococcal vaccinations as part of routine SCD standard-of-care or given right before eculizumab and continued on a prophylactic antibiotic regimen while on treatment.

CM-TMA from an underlying mutation involving the complement regulatory genes is traditionally called atypical hemolytic uremic syndrome or 'atypical HUS' (aHUS). CM-TMA occurring secondary to complement amplifying disorders such as hematopoietic stem cell transplantation, malignancy, infections, or autoimmune diseases are termed 'secondary HUS'.,4 The above clinical vignettes suggest that some complications of SCD may also reflect complement activation-induced secondary HUS (Figure 2). As SCD is a chronic hemolytic condition and plasma free HGB and heme can activate complement, ${ }^{5,6}$ additional complement activation during episodes of disease exacerbation may lead to increased hemolysis and a sustained positive feedback loop that leads to life-threatening anemia. As sickle erythrocytes are uniquely susceptible to complement-mediated damage, ${ }^{7}$ this may also result in further exacerbation of the disease. Heme-dependent endothelial dysfunction seen in SCD can also be modulated by complement activation..$^{8}$ In addition, heme can regulate the coagulation system, which likely reflects another important feature of SCD pathophysiology that contributes to inflammation and thrombosis. ${ }^{9}$ Increased C5a production has also been shown to cause acute lung injury and vasoocclusion in animal models. ${ }^{10,11}$ Prior reports suggested the role of ACP in the pathophysiology of SCD, and a protective effect of eculizumab in some settings. ${ }^{12-15}$ However, the present data suggest that some complications in SCD may not only reflect exuberant ACP activation, but actually represent an underlying CM-TMA. In these situations, chronic hemolysis and endothelial dysfunction may saturate scavenging and detoxifying mechanisms, reducing the capacity of patients with SCD to manage elevated plasma HGB and heme during periods of crises. In two of our patients, we detected variants in the complement genes. The CFH gene encodes soluble plasma factor $\mathrm{H}$, which is a principal inhibitor of ACP. Further genetic studies focusing on complotype are needed to help understand its role in SCD. Figure 2 compares salient clinical features seen in aHUS and CM-TMA in SCD. In this way, the underlying pathophysiology of SCD may prime individuals for secondary HUS through ACP activation, with a subset of these patients reaching an inflection point during periods of crises (second-hit) that lead to additional hyperhemolysis and MOF. Case \#3 is the first report of successful use of eculizumab to treat life-threatening DIIHA. Ceftriaxone-induced hemolysis often occurs secondary to immunglobulin M (IgM) anticeftriaxone antibodies, which typically engage the classical complement pathway and induce hemolysis. However, the impact of eculizumab did not appear to reflect inhibition of IgM-induced hemolysis as marked activation of APC was evidenced by increased levels of factor $\mathrm{Bb}$, which likewise responded to eculizumab, consistent with a positive feedback loop that drives additional APC activation in these patients. While rare, this event in particular holds public health and preventative importance, as ceftriaxone is a widely used medication in SCD, and there is a high prevalence of ceftriaxone-induced $\mathrm{RBC}$ antibodies in these patients. ${ }^{16}$

This collection of cases therefore emphasizes a previously underappreciated role of CM-TMA and complement across a broad range of SCD presentations, and may reflect novel insights into the pathophysiology of acute exacerbations of SCD that may be sensitive to complement inhibition to avoid severe hemolytic complications in SCD.

Satheesh Chonat, ${ }^{1,2}$ Sara Graciaa, ${ }^{2}$ H. Stella Shin, ${ }^{1,3}$ Joanna G. Newton, ${ }^{1,2}$ Maa-Ohui Quarmyne, ${ }^{1,2}$ Jeanne Boudreaux, ${ }^{1,2}$ Amy Tang, ${ }^{1,2}$ Patricia E. Zerra, ${ }^{2,4}$ Margo R. Rollins, 2,4 Cassandra D. Josephson, 2,4 Clark Brown, ${ }^{1,2}$ Clinton H. Joiner, ${ }^{1,2}$ Ross M. Fasano ${ }^{2,4}$ and Sean R. Stowell

'Department of Pediatrics, Emory University School of Medicine; ${ }^{2}$ Aflac Cancer and Blood Disorders Center; ${ }^{3}$ Division of Pediatric Nephrology, Children's Healthcare of Atlanta and ${ }^{4}$ Center for Transfusion and Cellular Therapy, Department of Pathology and Laboratory Medicine, Emory University School of Medicine, Atlanta, GA, USA.

Correspondence:

SATHEESH CHONAT - satheesh.chonat@emory.edu

doi:10.3324/haematol.2020.262006

Acknowledgments: the authors would like to thank the hematology, critical care medicine, nephrology and transfusion medicine teams at Children's Healthcare of Atlanta for their assistance with patient care. No funding was used to conduct this study.

\section{References}

1. Vidler JB, Gardner K, Amenyah K, Mijovic A, Thein SL. Delayed haemolytic transfusion reaction in adults with sickle cell disease: a 5-year experience. Br J Haematol. 2015;169(5):746-753.

2. Habibi A, Mekontso-Dessap A, Guillaud C, et al. Delayed hemolytic transfusion reaction in adult sickle-cell disease: presentations, outcomes, and treatments of 99 referral center episodes. Am J Hematol. 2016;91(10):989-994

3. Riedl M, Fakhouri F, Le Quintrec M, et al. Spectrum of complementmediated thrombotic microangiopathies: pathogenetic insights identifying novel treatment approaches. Semin Thromb Hemost. 2014;40(4):444-464

4. Le Clech A, Simon-Tillaux N, Provôt F, et al. Atypical and secondary hemolytic uremic syndromes have a distinct presentation and no common genetic risk factors. Kidney Int. 2019;95(6):1443-1452.

5. Frimat M, Tabarin F, Dimitrov JD, et al. Complement activation by heme as a secondary hit for atypical hemolytic uremic syndrome. Blood. 2013;122(2):282-292.

6. Merle NS, Grunenwald A, Rajaratnam H, et al. Intravascular hemolysis activates complement via cell-free heme and heme-loaded microvesicles. JCI Insight. 2018;3(12):1-18.

7. Wang RH, Phillips G, Medof ME, Mold C. Activation of the alternative complement pathway by exposure of phosphatidylethanolamine and phosphatidylserine on erythrocytes from sickle cell disease patients. J Clin Invest. 1993;92(3):1326-1335.

8. Merle NS, Paule R, Leon J, et al. P-selectin drives complement attack on endothelium during intravascular hemolysis in TLR-4/heme- 
dependent manner. Proc Natl Acad Sci U S A. 2019;116(13):62806285.

9. Markiewski MM, Nilsson B, Nilsson Ekdahl K, Mollnes TE, Lambris JD. Complement and coagulation: strangers or partners in crime? Trends Immunol. 2007:28(4):184-192.

10. Mulligan MS, Schmid E, Beck-Schimmer B, et al. Requirement and role of $\mathrm{C} 5 \mathrm{a}$ in acute lung inflammatory injury in rats. J Clin Invest. 1996;98(2):503-512.

11. Vercellotti GM, Dalmasso AP, Schaid Jr TR, et al. Critical role of C5a in sickle cell disease. Am J Hematol. 2019;94(3):327-337.

12. Chonat S, Chandrakasan S, Kalinyak KA, Ingala D, Gruppo R, Kalfa TA. Atypical haemolytic uraemic syndrome in a patient with sickle cell disease, successfully treated with eculizumab. Br J Haematol. 2016;175(4):744-747.
13. Dumas G, Habibi A, Onimus T, et al. Eculizumab salvage therapy for delayed hemolysis transfusion reaction in sickle cell disease patients. Blood. 2016;127(8):1062-1064.

14. Chonat S, Quarmyne M-O, Bennett CM, et al. Contribution of alternative complement pathway to delayed hemolytic transfusion reaction in sickle cell disease. Haematologica. 2018;103(10):e483-e485

15. Thein SL, Pirenne F, Fasano RM, et al. Hemolytic transfusion reactions in sickle cell disease: underappreciated and potentially fatal. Haematologica. 2020;105(3):539-544.

16. Quillen K, Lane C, Hu E, Pelton S, Bateman S. Prevalence of ceftriaxone-induced red blood cell antibodies in pediatric patients with sickle cell disease and human immunodeficiency virus infection. Pediatr Infect Dis J. 2008;27(4):357-358. 